\title{
Application of CRISPR/cas9-Directed Homologous Recombination to the Generation of Human Tumor Cells with Conditional Knockout of an X-Linked MicroRNA Locus
}

\author{
Aurélie Van Tongelen ${ }^{1}$, Axelle Loriot ${ }^{1}$, Olivier De Backer ${ }^{2}$ and Charles De Smet ${ }^{1}$ \\ ${ }^{1}$ Department of Genetics and Epigenetics, de Duve Institute, Université catholique de Louvain, Brussels, Belgium \\ ${ }^{2}$ URPhyM NARILIS, University of Namur-FUNDP, Namur, Belgium
}

\begin{abstract}
Studying the cellular function of microRNAs requires genetic strategies to generate their loss-of-function. Recently, a novel approach of targeted genomic deletion was proposed, which is based on induction of site-specific DNA cuts with Cas9/gRNA ribonucleoprotein complexes, combined with homologous recombination-dependent insertion of cassettes that contain sequences for Cre-lox or FLP-FRT systems. Here, we provide a technical report describing application of this CRISPR/Cas9-directed homologous recombination procedure to the generation of human tumor cells in which conditional knockout of an X-linked cluster of microRNAs (miR-105/miR-767) can be induced. We describe the successive steps of genetic engineering and cell clone selection that allowed us to generate cells with the expected genome editing.
\end{abstract}

Keywords: CRISPR/cas9; MicroRNA; FLP recombinases; LoxP; Dulbecco's medium; T4 DNA ligase

\section{Introduction}

MicroRNAs (miRNAs) are small noncoding RNAs that exert important cellular functions by repressing post-transcriptional gene expression through binding to target mRNAs [1]. More than $60 \%$ of all human genes are predicted to be regulated by a total of over 2.000 mature miRNAs. Some miRNAs are expressed in virtually all cell types, whereas others are highly tissue-specific with a distinct function depending on cell type or organ. miRNAs play key roles in most biological processes, including cell division and death, cellular metabolism, intracellular signaling, immunity and cell movement. Not surprisingly, disregulation of miRNA expression has been functionally linked to various pathological and occasionally malignant outcomes [2]. Specific miRNA expression patterns, which have been associated with particular diseases, hold great prognostic value. In cancer, oncogenic miRNAs as well as tumor suppressor miRNAs have been identified, and therapeutic strategies directed against such cancer-related miRNA are being considered $[3,4]$.

The physiological roles of most miRNAs still need to be deciphered. Therefore, tools allowing manipulation of miRNA activity are required. Gain-of-function by over-expression of the miRNA is relatively easy, and can be achieved by transfection of synthetic microRNAs or by enforcing expression of primary miRNA transcripts [5,6]. Lossof-function analyses are less obvious. miRNA inhibitors based on synthetic antisense molecules or miRNA sponges, which act by binding and sequestering miRNAs away from their natural targets, are available [7]. However, such inhibitors have some disadvantages, including incomplete masking of miRNA function, and uncontrolled off-target effects.

Genome engineering is a powerful tool for dissecting biological mechanisms. The Clustered, Regularly Interspaced, Short Palindromic Repeats (CRISPR) / CRISPR-associated protein 9 (Cas9) system provides a rapid and efficient technology for targeted genome editing. In association with a specifically designed guide RNA (gRNA), Cas9 can achieve site-specific DNA recognition and cleavage [8]. A constraint in this genome editing process is the compulsory presence of a Protospacer Adjacent Motif (PAM) sequence near the Cas9 cleavage site. In most cases, the site-specific DNA double-strand breaks (DSB) induced by Cas9 triggers a non-homologous end-joining (NHEJ) process of DNA repair, leading to small insertions or deletion in the nucleotidic sequence. This procedure is therefore exploited to generate loss-of-function of protein coding genes, via alteration of the open reading frame. Short miRNAs are less amenable to this editing process, as many of them do not carry the required PAM motif within their sequence.

An alternative use of the CRISPR/Cas9 technology, which would be better adapted to manipulate miRNAs, has been proposed. In this setting, Cas9 cleavages are used to direct homologous recombinationdependent insertion of cassettes that contain LoxP or FRT sequences up- and downstream of the miRNA locus, thereby allowing subsequent deletion of the embedded sequence upon expression of Cre or FLP recombinases. This procedure requires that template DNA sequences with homologous arms are provided to the cells, in order to stimulate homology-directed repair (HDR) of the Cas9-iduced DSB [9]. Examples where this technology was used to delete a miRNA locus remain however scarce $[10,11]$. Here, we report successful application of this procedure to the generation of human tumor cells in which a miRNA locus can be conditionally deleted. The locus targeted in our study corresponds to an X-linked cluster of two miRNAs (miR-105 and miR-767), which were recently shown to display aberrant activation in wide variety of tumors, and to exert oncogenic potential $[12,13]$.

\section{Materials and Methods}

\section{Cell culture and transfection}

HT1080, LB2201-MEL, Mi13443, SK-MES1, GLCP1 cell lines were cultured in Iscove's modified Dulbecco's medium (IMDM, Life

*Corresponding author: Charles De Smet, de Duve Institute, Université catholique de Louvain, Brussels, Belgium, Tel: 322.764.7523; E-mail: charles. desmet@uclouvain.be

Received July 01, 2015; Accepted July 17, 2015; Published July 20, 2015

Citation: Tongelen AV, Loriot A, De Backer O, De Smet C (2015) Application of CRISPR/cas9-Directed Homologous Recombination to the Generation of Human Tumor Cells with Conditional Knockout of an X-Linked MicroRNA Locus. Gene Technology 4: 124. doi: 10.4172/2329-6682.1000124

Copyright: @ 2015 Tongelen AV, et al. This is an open-access article distributed under the terms of the Creative Commons Attribution License, which permits unrestricted use, distribution, and reproduction in any medium, provided the original author and source are credited. 
Citation: Tongelen AV, Loriot A, De Backer O, De Smet C (2015) Application of CRISPR/cas9-Directed Homologous Recombination to the Generation of Human Tumor Cells with Conditional Knockout of an X-Linked MicroRNA Locus. Gene Technology 4: 124. doi: 10.4172/2329-6682.1000124

Page 2 of 8

Technologies) and HEK293 cell line in 4,5 g/l D-glucose Dulbecco's modified Eagle's medium (DMEM, Life Technologies). All media were supplemented with $10 \%$ fetal bovine serum (FBS, Life Technologies) and contained $1 \mathrm{x}$ non-essential amino acids (Life Technologies) and $1 \mathrm{x}$ Penicillin/Streptomycin (Life Technologies).

DNA transfection was performed in a T75 flasks, with 2 to 6 millions cells, using Lipofectamine $2000^{\circ}$ Reagent (Invitrogen). Five hours after transfection, the medium was replaced. The amount of transfected DNA was $5 \mu \mathrm{g}$ of Cas9/sgRNAs vectors and $5 \mu \mathrm{g}$ of donor pEZ-Frt-lox-5' or pEZ-Frt-lox-3' vectors. Prior to transfection, pEZFrt-lox-5' vector was linearized 5' to the left homology arm by digestion with the EcoRV restriction enzyme, and the pEZ-Frt-lox-3' vector was digested by EcoRV and XhoI restriction enzymes. Transfectants were selected for 15 days in medium containing 0.8 to $2 \mathrm{mg} / \mathrm{ml}$ of geneticin, depending on the type of cells. Two weeks after transfection, integration of the donor DNA was tested by PCR amplification with primers encompassing the recombinant fragment on genomic DNA. Cell populations showing effective recombination were cloned by limiting dilutions.

For Cre or FLP recombination, cells were transiently transfected using Lipofectamine $2000^{\circ}$ Reagent (Invitrogen) in 6-well plates with $2 \mu \mathrm{g}$ of CMV-Cre or pCAGGS-FLPe vectors, and cloned by limiting dilution $72 \mathrm{~h}$ after transfection.

\section{Plasmid design and construction}

The pX330-U6-Chimeric_BB-CHb-hSpCas9 vector (available at Addgene, and kindly provided by P. Coulie) was digested using FastDigest BbsI (Thermo Scientific) and double-stranded oligonucleotides corresponding to different spacer sequences were ligated with T4 DNA ligase (Thermo Scientific) generating different sgRNAs. Double-stranded oligonucleotides for spacer sequences were obtained by annealing pairs of complementary oligos (Eurogentec) composed of the spacer sequence flanked by adapter sequences (Forward: 5'-CAACG...-3'; Complement: 5'-AAAC...C-3'). Design of spacer sequences was based on CRISPR Design Tool of ZhangLab (http://crispr. mit.edu) generating: sgRNA1 5' -TTTGTGTAATGGGATCGTTTGG; sgRNA2, 5'-GGGGATAGATATGGTATCCCAGG; sgRNA3 5'-CAATCATTGTCTACTTGTACTGG; sgRNA4 5'-CTGCAACATGAGCTGATACCAGG; sgRNA5 5'- TGGTAGACTGAGCACGCGTAAGG; sgRNA6 5'- GGTCGTCTGATGTTATACCTTGG; sgRNA7 5'- AGGAATTCTTTCCCGTATTGTGG; sgRNA8 5'- TACGCGTGCTCAGTCTACCATGG.

Donor vector constructions were performed on the basis of the pEZ-Frt-lox-DT vector (Addgene). Left (LHA) and right (RHA) homology arms ( $\sim 300$ to $\sim 800 \mathrm{pb})$ were amplified by PrimeSTAR HS DNA Polymerase (Takara) from HT1080 genomic DNA using complementary primers flanked by restriction site sequence at the 5 ' end. For the pEZ-Frt-lox-5' vector, primers to amplify LHA were flanked with EcoRV and NotI sites (sense: 5-attaGATATCtaggcatgtgttcatccgagga; reverse: 5'-attaGCGGCCGCgatcccattacacaaactaacagc), and primers to amplify RHA were flanked with HindIII and XhoI sequences (sense: 5'-attaAAGCTTgtttggatttatatcatgcccaaac; reverse: 5'-attaCTCGAGgataccaggttatagggagtaga). For the pEZ-Frt-lox-3' vector, primers to amplify LHA were flanked with EcoRV and NotI sites (sense: 5'-attaGATATCgtgtatgtgcacaatgctcact; reverse: 5'-attaGCGGCCGCccatggagaatgagccaatact), and primers to amplify RHA were flanked with SalI and XhoI sequences (sense: 5'- attaGTCGACtagactgagcacgcgtaaggaa; reverse: 5'-attaCTCGAGccatagactggtacttaccgaga). PCR products were digested with the appropriate restriction enzymes, and purified (QIAquick Gel Extraction Kit, Qiagen), prior to ligation with T4 DNA ligase
(Thermo Scientific) into the pre-digested vector. pEZ-Frt-lox-DT vector was pre-digested with EcoRV-NotI for the insertion of both LHA, and with HindIII-XhoI or SalI-XhoI for introduction of RHA to pEZFrt-lox-5' or pEZ-Frt-lox-3' vectors, respectively. All PCR products and final vectors were verified by DNA sequencing.

\section{DNA preparation and PCR}

Two weeks after cloning, HT1080 clones were removed from 96-well plates with $2 \mathrm{mM}$ EDTA/PBS. Half of the cells were reseeded in 24-well plate and the other half was used for DNA extraction. Cells were placed in a lysis buffer composed by $10 \mathrm{mM}$ Tris $\mathrm{pH} 8,10 \mathrm{mM}$ EDTA, $1 \%$ SDS and $0.1 \mathrm{mg} / \mathrm{ml}$ of proteinase $\mathrm{K}$ (New England BioLabs). After overnight incubation at $45^{\circ} \mathrm{C}$, DNA was extracted by organic extraction with phenol:chloroform and $1 \mu \mathrm{g}$ of salmon sperm DNA was added to help isopropanol precipitation. PCR S1-4 (Forward: 5'-gtcactggctcctgcagaat; reverse: 5'-gaagcagatggcaatgttgcaa), PCR S5-8 (Forward: 5'-gagaatgtctggttgtgtacctt; Reverse: 5'-caggcaaacacgatactgtcaa), PCR A (Forward: 5'-cactgtcatcttatccttaactga; Reverse: 5'-ctcgaccatatgggagagct), PCR B (Forward: 5' -gacgagttcttctgagggga; Reverse: 5'-atttgaccacgatgcacacaca), PCR C (Forward: 5'-ggctcctgcagaatgggtat; Reverse: 5'-acacctacaggacataggctat), PCR D (Forward: 5'-atatgaggaagcatggcaccat; Reverse: 5'-ctcgaccatatgggagagct), PCR E (Forward: 5'-gacgagttcttctgagggga; Reverse: 5'-gataactatggtgatgcacataca), PCR F (Forward: 5'-atatgaggaagcatggcaccat; Reverse: 5'-gataactatggtgatgcacataca), PCR G (Forward: 5'-ggctcctgcagaatgggtat; Reverse: 5'-tgctacctctaatggatatggtaa), PCR 105 (Forward: attctcgagccctttagctatggtcttctgct; Reverse: gatacgcgttgatggtgccatgcttcctca) and PCR 767 (Forward: 5'-attctcgagtttaacagtcaaatattagtgttagttgct; Reverse: 5'-gatacgcgtcagccatcacatcataggca) were performed using Dream Taq polymerase (Thermo Scientific) on 1/3 of the total extracted DNA.

DNA extracted from total cell populations was performed similarly, but without addition of salmon sperm DNA. DNA concentrations were quantified with a NanoDrop ND-1000 (NanoDrop Technologies), and PCR were performed on $100 \mathrm{ng}$ of genomic DNA per reaction.

\section{RNA preparation and RT-qPCR evaluation of miRNA expression levels}

We isolated total RNA using TriPure Isolation Reagent (Roche) according to the manufacturer's protocol, and used 100ng of this RNA for reverse transcription with the miRCURY LNATM Universal RT microRNA PCR (Exiqon). The resultant CDNA was diluted 20 times, and a $2.5 \mu \mathrm{l}$ aliquot was used in a SYBRGREEN qPCR with LNA specific primers for miR-105 and miR-767 (Exiqon). RNU44 was used as internal control.

\section{Flow cytometry cell sorting}

HT1080 \#72.2.25.9 clone was transiently transfected by pTM945Cre-IRES-mCherry vector or control vector pTM945-mCherry, coding both for mCherry fluorescent protein. Two days later, cells were sorted by fluorescence-activated cell sorting (FACS, FACSAriaTM III, BD Biosciences) based on mCherry signal. The sorted populations were harvested for RT-qPCR analysis 6 to 34 days after reseeding.

\section{Detection of gRNA efficiency}

HT1080 cells were transfected with pX330-U6-Chimeric_BB-CBhhSpCas9 vector carrying 8 different sgRNAs, named Cas9/sgRNA1-8. Three days after transfection, DNA extracted from those cells was treated with RNAse A and submitted to SURVEYOR ${ }^{\circ}$ Mutation Detection Kit, according to the manufacturer's recommendations (Transgenomic). In brief, the genomic region flanking the CRISPR/Cas9 target site was first 
amplified by PCR using PrimeSTAR HS DNA polymerase (Takara). After migration through agarose gel, PCR products were purified using QIAquick Gel Extraction Kit (Qiagen). $2 \mu \mathrm{g}$ of the purified PCR products were mixed with 10x Surveyor Buffer (100 mM Tris, $500 \mathrm{mM}$ $\mathrm{KCl}, 15 \mathrm{mM} \mathrm{MgCl}_{2}$ ) and water to a final volume of $20 \mu \mathrm{l}$, and subjected to a re-annealing process to enable heteroduplex formation: $95^{\circ} \mathrm{C}$ for $10 \mathrm{~min}, 95^{\circ} \mathrm{C}$ to $85^{\circ} \mathrm{C}$ ramping at $-2^{\circ} \mathrm{C} / \mathrm{s}, 85^{\circ} \mathrm{C}$ to $25^{\circ} \mathrm{C}$ at $-0.25^{\circ} \mathrm{C} / \mathrm{s}$, and $25^{\circ} \mathrm{C}$ hold for 1 minute. After re-annealing, PCR products were treated with $1 \mu \mathrm{l}$ of SURVEYOR nuclease and SURVEYOR enhancer $\mathrm{S}$ (Transgenomics) during 1 hour at $42^{\circ} \mathrm{C}$. DNA samples were run in a $2 \%$ agarose gel, in TAE $1 \mathrm{x}$ buffer supplemented with ethidium bromide. Quantification was performed by calculating relative band intensities using ImageJ software.

\section{Results and Discussion}

\section{General strategy for the generation of cells with conditional knockout of MIR105/767}

The general strategy to obtain cells where the X-linked MIR105/767 locus can be conditionally deleted is described in Figure 1 and each step is described in details below. In brief, the procedure involved successive steps of CRISPR/cas9-directed homologous recombination to permit integration of neomycin-loxP/FRT cassettes 3 ' and 5 ' to the MIR105/767 genomic locus, as well as exposure of cells to Cre and FLP
(Flippase) recombinases to leave only single loxP sites on both sides. At each step of the procedure, a cell clone with the appropriate integration/ recombination was selected, and used for the subsequent step.

\section{Selection of efficient single guide RNAs (sgRNAs)}

Targeting of the Cas9 nuclease to a specific site on the genome is commonly achieved through its association with a chimeric RNA construct, termed single guide RNA (sgRNA). The sgRNA includes the tracrRNA sequence, which allows association of the RNA molecule with the Cas9 enzyme, and the spacer sequence, which binds the genomic target by nucleotide complementarity. Not all sgRNAs are equally efficient. The first step to achieve our construction was therefore to test different sgRNAs, and to select those that stimulate site-specific cleavage by Cas9 with the highest efficiency (Figure 2). Using a spacer RNA design algorithm (http://crispr.mit.edu), we designed four sgRNAs for each side of the MIR105/767 locus. The corresponding sequences were inserted in the pX330-U6-Chimeric_BB-CBh-hSpCas9 vector, which also carries a human codon-optimized Cas 9 coding sequence. These different Cas9/sgRNA vectors were then transfected into HT1080 cells, generating DSBs at precise sgRNA targeted sites. The error-prone NHEJ process results in the formation of mutation or small insertions or deletion. Genomic DNA was collected from transfectants after three days. DNA cleavage efficiency was evaluated by mutation detection assay using the Surveyor ${ }^{\circledR}$ Mutation Detection kit. Thus, DNA

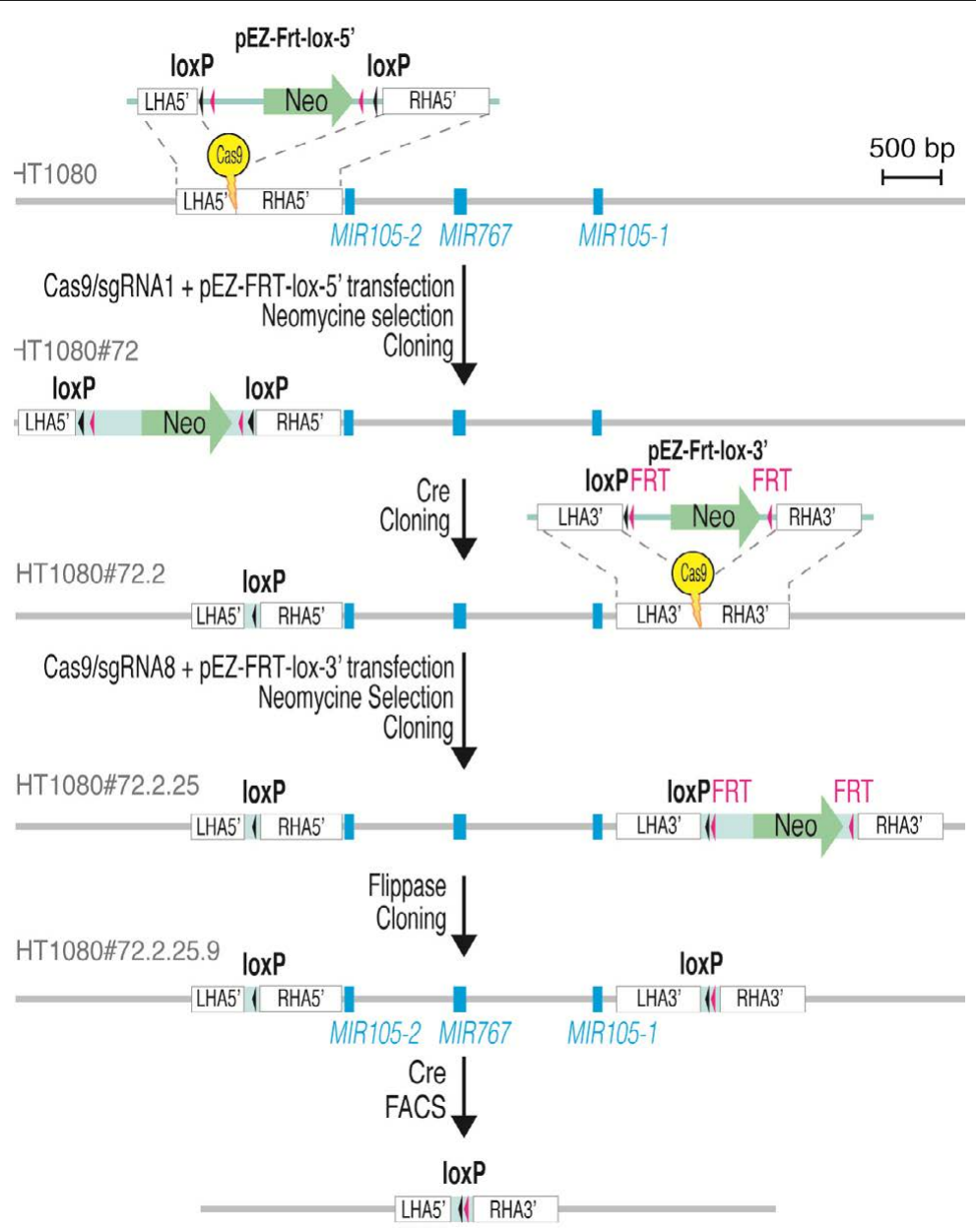

The MIR105/767 locus comprises two pre-microRNA sequences (MIR105-1 and MIR105-2) for miR-105 and one (MIR767)for miR-767, represented by blue boxes. Refer to the text for detailed explanation on the targeting vectors, and the successive steps of cell transfection and clone selection.

Figure 1: Schematic draw depicting the strategy used for generation of a cell line with a "floxed" MIR105/767 locus. 


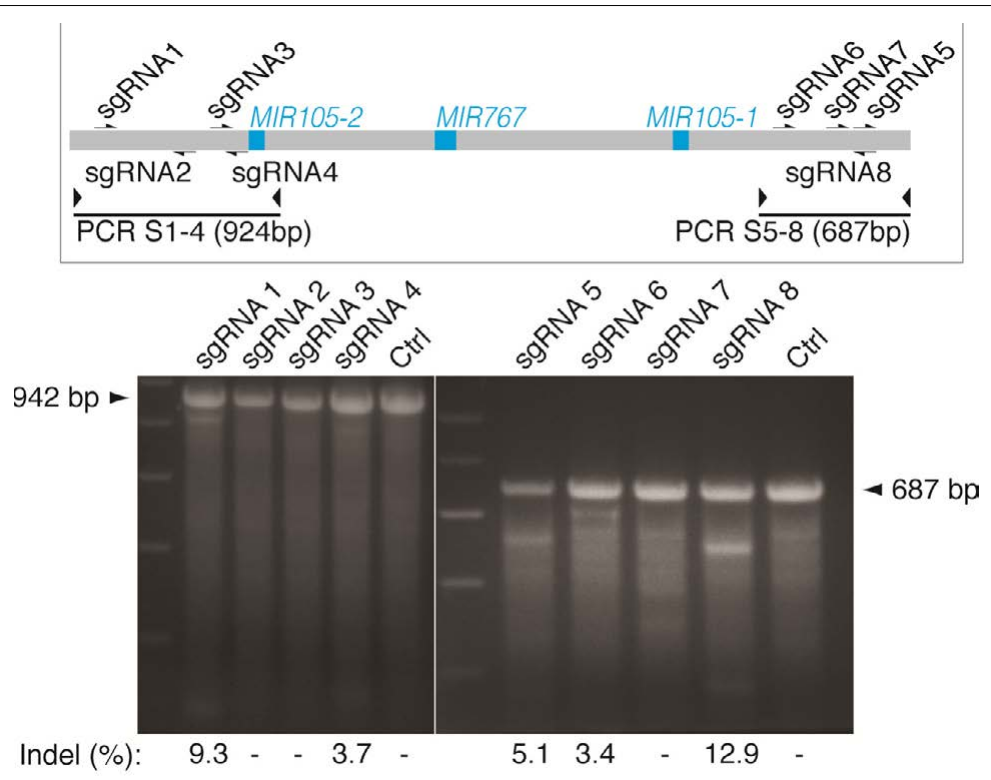

On the top panel, the schematic draw depicts localisation of each sgRNA on the MIR105/767 locus, as well as the position of primers used for PCR amplification prior to the Surveyor nuclease treatment. Sizes of initial (uncleaved) PCR products are also given. Transfections with each of the Cas9/sgRNA vectors (sgRNA1 to -8) were performed in HT1080 cells, and DNA was extracted from transfectants 3 days later. DNA samples were submitted to PCR amplification with indicated primers, and then exposed to Surveyor nuclease. Resulting DNA products were analyzed by gel electrophoresis. Presence of DNA fragments with a size lower than the original PCR product indicates efficient sgRNA-directed cleavage. The ratio between the intensity of the lower band and that of the starting PCR band provides a measurement of cleavage efficiency (\% indel).

Figure 2: Surveyor assay comparing the cleavage efficiency induced by eight different sgRNAs at corresponding DNA regions.

fragments carrying the expected cleavage site were amplified by PCR. Amplicons were denatured by heat, and then allowed the re-anneal slowly. They were then exposed to the Surveyor nuclease, which cuts heteroduplexes but not homoduplexes of DNA. Nuclease-treated DNA fragments were electrophoresed in an agarose gel, and the cleavage efficiency was evaluated by calculating the ratio between the cleaved and uncleaved band (percentage of indel). Using this method, we identified the sgRNAs that directed the most efficient Cas9-mediated cleavage either 5' (sgRNA1, 9.3\% indel) or 3' (sgRNA8, 12.9\% indel) to the MIR105/767 locus (Figure 2).

\section{CRISPR/Cas9 mediated integration of a Neo-2loxP/2FRT} cassette 5' to the MIR105/767 locus

In order to direct homologous recombination towards the sgRNA1induced Cas9 cleavage site, a vector (pEZ-FRT-lox-5') was constructed in which left and right homology arms were inserted on each side of a neomycin resistance cassette (Neo) flanked by two loxP and FRT sites (Neo-2loxP/2FRT; Figure 3). The left homology arm (LHA5, 306bp) and the right homology arm (RHA5, 692bp) corresponded to genomic sequences located upstream and downstream the Cas9/sgRNA1 cutting site, respectively.

We transfected Cas9/sgRNA1 and pEZ-FRT-lox-5' vectors in six different human cell lines: embryonic kidney cells (HEK293), two melanoma cell lines (Mi13443 and LB2201-MEL), two lung carcinoma cell lines (GLCP1 and SKMES1), and a fibrosarcoma cell line (HT1080). We selected cells of male origin, as these contain only one X chromosome. Following transfection, cells were selected for two weeks in neomycin-containing medium, and part of the resistant populations was harvested for DNA extraction. Integration of the Neo-2loxP/2FRT cassette was assessed by PCR amplification with one primer matching a genomic sequence upstream of the LHA5' and another primer corresponding to a sequence within the cassette. Of the six transfected cell lines, only three (HT1080, GLCP1 and SK-MES1) showed evidence of integration of the Neo-2loxP/2FRT cassette (Figure 3).

We decided to derive clones from the transfected HT1080 cell population, in order to isolate clones harboring the appropriate integration event. Out of 113 clones, 4 showed integration of the Neo-2loxP/2FRT, as evidenced by positive signals following PCR amplification with appropriate primers (Figure 4). Following verification of PCR products by sequencing, only clones \#72, \#76 and \#83 showed perfect recombination. The remaining clone (\#29) showed deletions in the intersection between homology arms and the cassette sequence. Overall, we observed a $2.5 \%$ (3/113) efficiency of homologous recombination directed by CRISPR/Cas 9 towards the $5^{\prime}$ side of the MIR105/767 locus in HT1080 cells. Clone HT1080\#72 was selected for the subsequent steps.

\section{Cre recombinase-mediated removal of the 5' cassette}

In order to obtain cells where the Neo-2loxP/2FRT cassette was removed and only one loxP sequence remained 5' to the MIR105/767 locus, HT1080\#72 cells were transiently transfected with a vectors encoding Cre recombinase (CMV-Cre). Sub-clones were isolated, and screened for effective loxP recombination by PCR amplification with primers matching sequences in LHA5' and RHA5' homology arms (Figure 5). Out of five tested clones, two clones (\#72.2 and \#72.3) showed effective recombination. We chose clone HT1080 \#72.2 for the subsequent steps aiming at introducing a second loxP site 3 ' to the MIR105/767 locus.

\section{CRISPR/Cas9-directed integration of a Neo-1LoxP/2FRT cassette 3' to the MIR105/767 locus}

In HT1080\#72.2 cells, we next performed directed integration of a Neo-1loxP/2FRT cassette towards the sgRNA8-induced Cas9 cleavage site, located on the 3' side of the MIR105/767 locus. To this end, we constructed a vector (pEZ-FRT-lox-3') in which left and right homology arms were inserted on each side of the Neo-1loxP/2FRT 

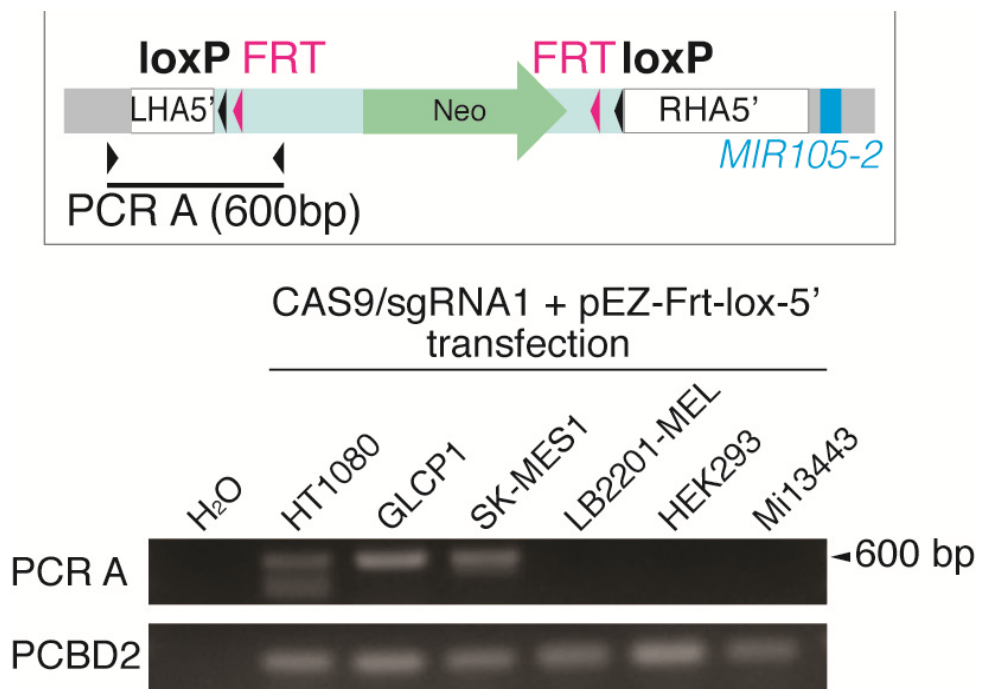

Following transfection, indicated cell lines were submitted to 2 weeks neomycin selection, and their genomic DNA was harvested for PCR analyses. The upper panel depicts the annealing sites of the PCR primers used to test integration of the Neo-2loxP/2FRT cassette (PCR A). One primer is located in the genomic region outside of the left homology arm (LHA5') and the other primer is located in the inserted sequence. Gel electrophoresis shows PCR amplification products obtained with primers for PCR A, and for the PCBD2, a gene located on chromosome 5 used as positive control.

Figure 3: PCR screening for insertion of Neo-2loxP/2FRT cassette in six cell lines transfected with pX330-sgRNA1 and pEZ-FRT-lox-5' vectors.

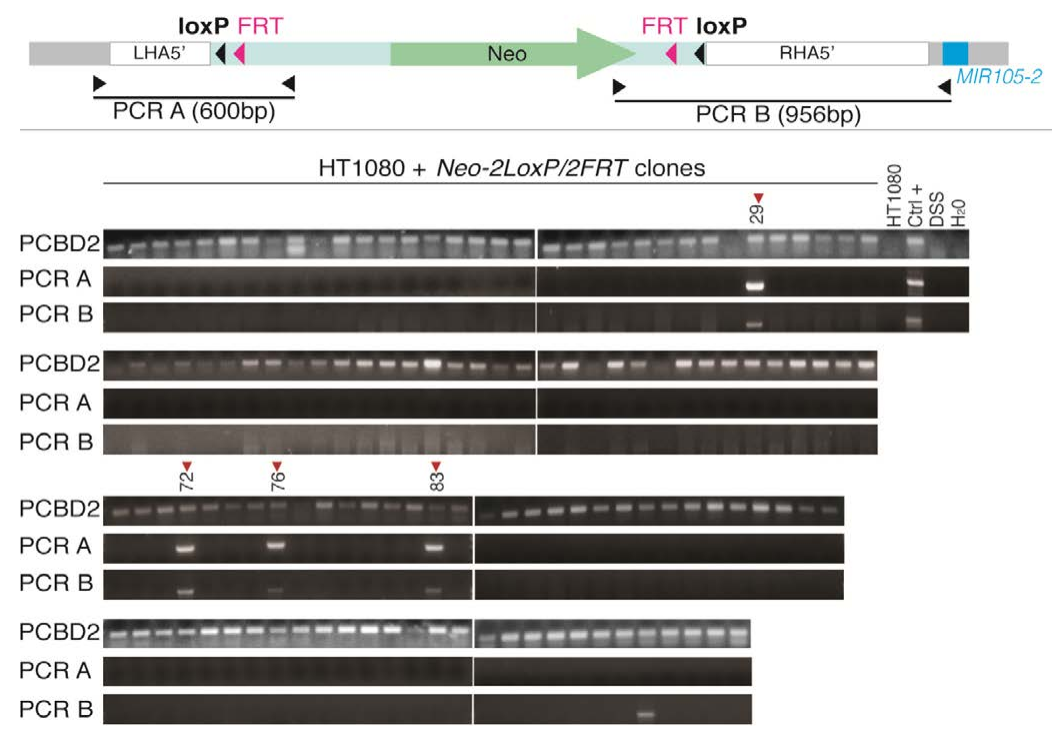

Following transfection with pX330-sgRNA1 and pEZ-FRT-lox-5' vectors, HT1080 cells were selected in neomycin, and 140 cell clones were derived. Their DNA was submitted to PCR analyses. The picture above depicts the annealing sites of the PCR primers used for screening clones with effective homologous integration of the Neo-2loxP/2FRT cassette. PCR A (600bp) and PCR B (956bp) have both one primer located in genomic DNA outside the homology arms, and another primer located in the donor vector sequence. Recombinant clones are expected to produce a signal with both PCRs. PCR amplification of $P C B D 2$ served as control. According to PCBD2 amplification, only 113 of these were analyzable. Control samples included the total population of $\mathrm{HT} 1080$ cells transfected with pX330-sgRNA1/pEZ-FRT-lox-5' (HT1080 sgRNA1), untransfected HT1080 cells, salmon sperm DNA (DSS), and water.

Figure 4: PCR screening for Neo-2loxP/2FRT cassette insertion in HT1080 cell clones.

cassette (Figure 6). The left homology arm (LHA3', 774bp) and the right homology arm (RHA3', 692bp) corresponded to genomic sequences located upstream and downstream of the Cas9/sgRNA8 cutting site, respectively. Restriction sites used for insertion of the RHA3' into the vector were chosen in order to delete the neighboring loxP site.

HT1080\#72.2 cells were transfected with Cas9/sgRNA8 pEZFRT-lox-3' vectors, and following neomycin selection, resistant clones were derived. These clones were screened for integration of the Neo-
1loxP/2FRT cassette by PCR amplification on genomic DNA, using primer pairs matching either sequences within the cassette or genomic sequences outside of the homology regions (Figure 6). PCR signals of the expected size were detected in 4 out 33 tested clones, indicating that Cas9/sgRNA8-directed homologous recombination in HT1080\#72.2 cells was obtained with an efficiency of $12,1 \%$. Clone HT1080\#72.2.25 was selected for the subsequent steps.

\section{Flippase mediated deletion of the 3' cassette}



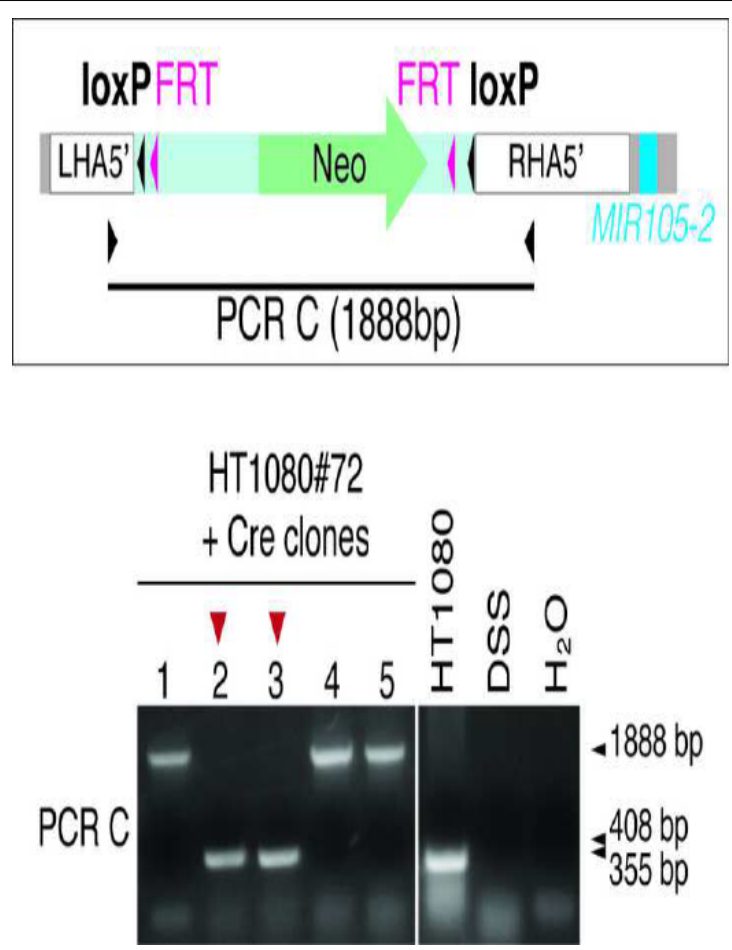

The schematic draw depicts the annealing sites of the PCR C primers used to detect "floxing" of the 5' cassette. The PCR product is expected at a size of $408 \mathrm{bp}$ in case of appropriate loxP recombination (1888bp in non-recombinant cells). PCR in naïve cells is expected to yield a $355 \mathrm{bp}$ product. Five clones isolated from the CMV-Cre-transfected HT1080\#72 cell population were tested. Control samples included untransfected HT1080 cells, salmon sperm DNA (DSS), and water.

Figure 5: PCR screening for Cre-induced loxP recombination at the 5' cassette.

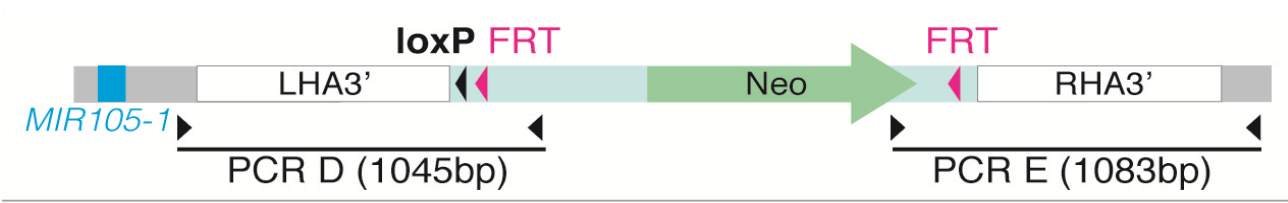

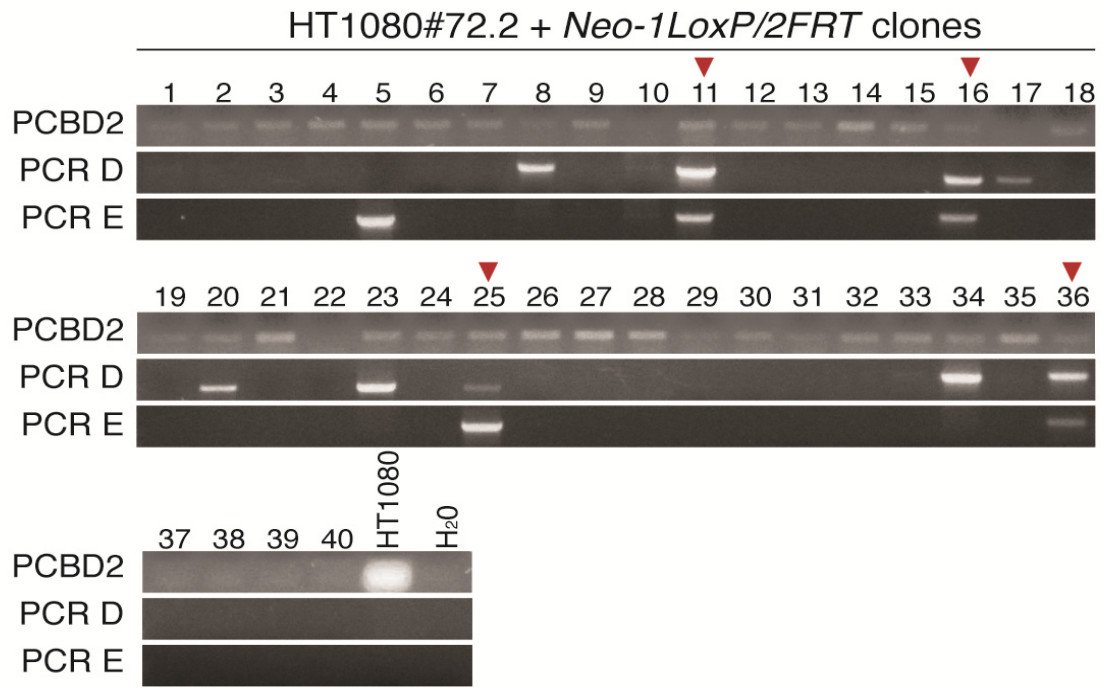

HT1080\#72.2 cells, which had been transfected with pX330-sgRNA8 and pEZ-FRT-lox-3' vectors, were selected in neomycin and cloned. DNA from 40 clones was tested for insertion of the Neo-1/oxP/2FRT cassette insertion 3' to the MIR105/767 by PCR. The upper panel indicates the position of the primers and expected product size of the two PCR (D and E). PCR results for the PCBD2 control sequence shows that only $33 / 44$ clones were analyzable. Control samples included untransfected HT1080 cells, and water.

Figure 6: PCR screening for Neo-1loxP/2FRT cassette insertion 3' to the MIR105/767 locus in HT1080 cell clones. 


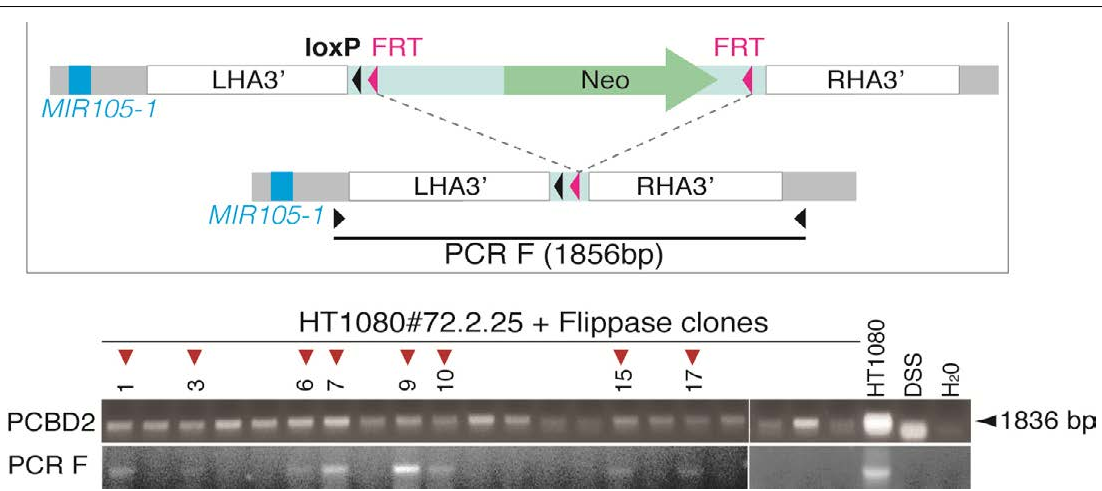

Clones were derived from HT1080\#72.2.25 that had been transiently transfected with a Flippase-encoding vector. DNA was extracted from 21 clones, and analyzed by PCR to detect FRT recombination. The schematic draw depicts the annealing sites of the PCR primers used for the screening (PCR F), and the expected PCR product size in case of effective FRT recombination. Amplification of PCBD2 served as positive control. Control samples included untransfected HT1080 cells, and water.

Figure 7: PCR screening for Flippase-induced FRT recombination at the 3' cassette.
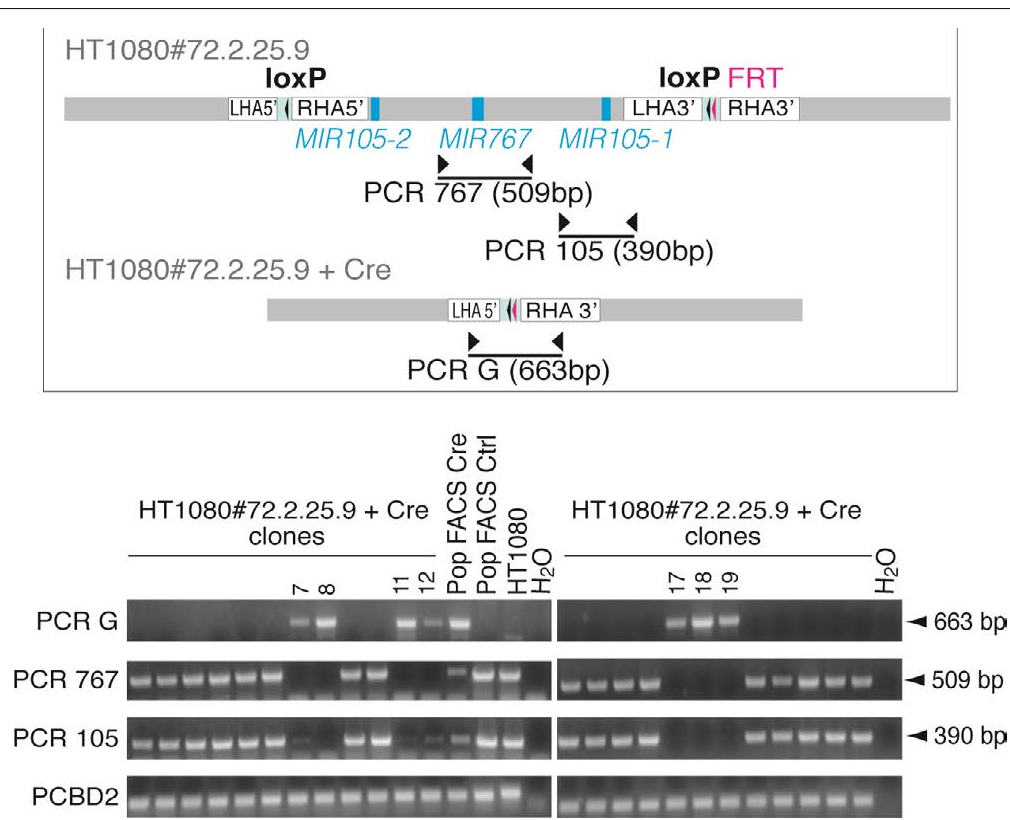

HT1080\#72.2.25.9 were transiently transfected with either CMV-Cre or pTM945-Cre-IRES-mCherry. CMV-Cre transfected cells were cloned, whereas pTM945-Cre-IRES-mCherry transfectants were sorted by FACS to isolate mCherry-expressing cells (Pop FACS Cre). A control population of cells was obtained after sorting cells that had been transfected with pTM945-mCherry (Pop FACS Ctrl). PCR screening was applied to cell clones ( $\mathrm{n}=24$ ) or sorted populations to detect deletion of the MIR105/767 locus. Three PCR amplifications were performed (PCR 767, PCR 105, PCR G). The upper panel indicates the position of PCR primers, and expected sizes of PCR products (following deletion for PCR G). Control samples included untransfected HT1080 cells, and water. Figure 8: PCR analysis of Cre-induced deletion of the MIR105/767 locus.

We next aimed to delete the cassette integrated 3' to the MIR105/767 locus, but without removing the single loxP site. To this end, HT1080\#72.2.25 cells were transiently transfected with a vector encoding Flippase, which induces recombination between FRT sites. Following transfection, cellular clones were isolated, and screened for effective FRT recombination by PCR amplification with primers matching sequences in LHA3' and RHA3' homology arms (Figure 7). Out of 21 tested clones, 8 clones (38\%) showed effective FRT recombination. Among these, clone HT1080 \#72.2.25.9, in which the MIR105/767 locus is now surrounded by two loxP sites, was chosen for subsequent analyses.

\section{Cre recombinase-mediated knockout of the MIR105/767} locus
Since HT1080\#72.2.25.9 cells contain loxP sequences on both sides of the MIR105/767 locus, their exposure to Cre recombinase is expected to induce deletion of the corresponding genomic region. To test this, HT1080\#72.2.25.9 cells were transiently transfected with the CMV-Cre vector. Previous experiments established a $40 \%$ efficiency of transfection in HT1080 cells, implying that only part of the transfected HT1080\#72.2.25.9 will effectively express Cre recombinase. We therefore decided to verify successful deletion of the MIR105/767 locus in clones isolated from the CMV-Cre transfected HT1080\#72.2.25.9 cell population. Following derivation of the clones, their DNA was extracted and submitted to PCR screening with three different pairs of primers allowing unambiguous determination of the presence or absence of the MIR105/767 locus (Figure 8). Among 24 tested clones, 7 


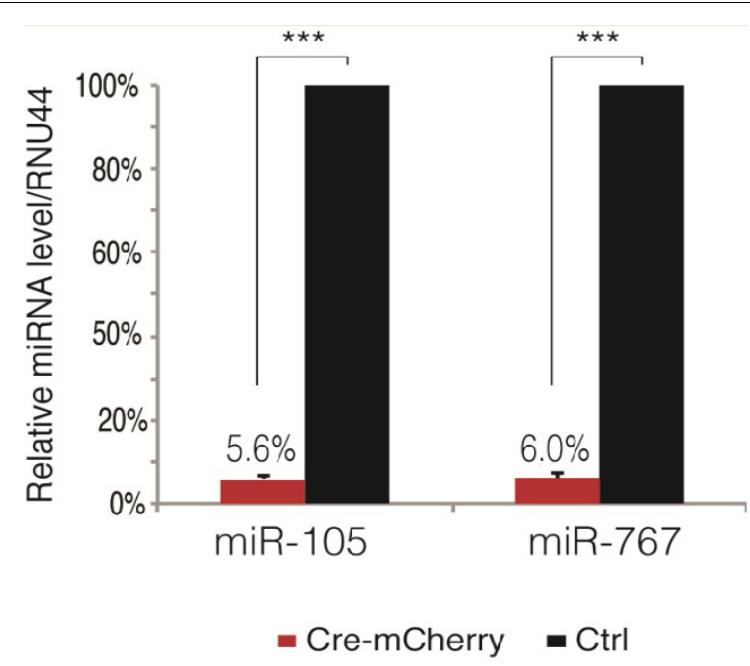

RNA was extracted from Cherry-expressing (FACS-sorted) HT1080\#72.2.25.9 cells that had been previously transfected with either pTM945-Cre-IRESmCherry (Cre-mCherry) or pTM945-mCherry (Ctrl). RT-qPCR analyses were performed at 6 different time points (days 6 to 34 ) post-transfection. Expression levels are expressed relative to the level in Ctrl cells. 2 way ANOVA was used for statistical analysis $\left.{ }^{* \star *}, p<0,0001\right)$

Figure 9: RT-qPCR expression analysis of miR-105 and miR-767 in FACSsorted Cre-induced HT1080\#72.2.25.9 cells.

clones (29\%) showed effective deletion of the MIR105/767 locus (Figure 8).

We next tried to facilitate the knockout procedure by avoiding the process of cellular cloning that followed CMV-Cre transfection. To this end, we replaced CMV-Cre with the pTM945-Cre-IRES-mCherry vector, which expresses both Cre recombinase and the mCherry fluorescent protein. Thus, HT1080\#72.2.25.9 cells were transfected with pTM945-Cre-IRES-mCherry, and two days later cells with the highest level of fluorescence were selected by fluorescence-activated cell sorting (FACS) and reseeded. A control group, transfected with a similar vector lacking Cre recombinase (pTM945-mCherry), was treated in parallel. Genomic DNA was extracted from FACS sorted cells, and submitted to PCR screening with the above-mentioned primer pairs (Figure 8 ). The results showed efficient enrichment of cells with MIR105/767 deletion, although a small proportion of non-recombined cells remained (Figure 8). We also analyzed the expression of miR-105 and miR-767 by RTqPCR in FACS-sorted cells. The results confirmed marked loss of expression of these two miRNAs in Cre-exposed cells, as only $5,6 \%$ and $6 \%$ residual expression level was observed for miR-105 and miR-767, respectively (Figure 9).

Altogether, we described a genetic engineering strategy based on CRISPR/Cas9-directed homologous recombination, which allowed us to create a human tumor cell line in which the MIR105/767 locus can be conditionally deleted. Our data therefore confirm the feasibility of this strategy, and provide several guidelines concerning the experimental procedures. CRISPR/Cas9-directed homologous recombination was found to occur with an expected efficiency ( $2.5 \%$ and $12.1 \%$ efficiency for the 5' and 3' position, respectively). One of the pitfalls of the CRISPR/ Cas9 technology is the presence of many off-target mutations induced by Cas 9 , which renders comparisons between genetically modified cells uncertain. This is however not a problem in our cellular model, as comparisons will be made within the final clone (HT1080\#72.2.25.9), between two cell groups that differ only for the presence or absence of the MIR105/767 locus.

\section{Acknowledgments}

This work was supported by grants from the D.G. Higher Education and Scientific Research of the French Community of Belgium (Action de Recherches Concertées) and from the Fonds special de recherche (FSR) of the Université catholique de Louvain, Belgium. A.V.T. is recipient of a Télévie grant from the FRSFNRS, Belgium [\#7.4581.13]. A.L. is supported by the de Duve Institute, Brussels, Belgium.

\section{References}

1. Bartel DP (2009) MicroRNAs: target recognition and regulatory functions. Cell 136: $215-233$.

2. Esteller M (2011) Non-coding RNAs in human disease. Nat Rev Genet 12: 861 874

3. Croce CM (2009) Causes and consequences of microRNA dysregulation in cancer. Nat Rev Genet 10: 704-714

4. Hayes J, Peruzzi PP, Lawler S (2014) MicroRNAs in cancer: biomarkers, functions and therapy. Trends Mol Med 20: 460-469.

5. Chen CZ, Li L, Lodish HF, Bartel DP (2004) MicroRNAs modulate hematopoietic lineage differentiation. Science 303: 83-86.

6. Wang $Z$ (2011) The guideline of the design and validation of MiRNA mimics Methods Mol Biol 676: 211-223.

7. Brown BD, Naldini L (2009) Exploiting and antagonizing microRNA regulation for therapeutic and experimental applications. Nat Rev Genet 10: 578-585

8. Mali P, Yang L, Esvelt KM, Aach J, Guell M, et al. (2013) RNA-guided human genome engineering via Cas9. Science 339: 823-826.

9. Cong L, Ran FA, Cox D, Lin S, Barretto R, et al. (2013) Multiplex genome engineering using CRISPR/Cas systems. Science 339: 819-823.

10. Ho TT, Zhou N, Huang J, Koirala P, Xu M, et al. (2015) Targeting non-coding RNAs with the CRISPR/Cas9 system in human cell lines. Nucleic Acids Res 43: e17.

11. Xiao A, Wang Z, Hu Y, Wu Y, Luo Z, et al. (2013) Chromosomal deletions and inversions mediated by TALENs and CRISPR/Cas in zebrafish. Nucleic Acids Res 41: e141.

12. Loriot A, Van Tongelen A, Blanco J, Klaessens S, Cannuyer J, et al. (2014) A novel cancer-germline transcript carrying pro-metastatic miR-105 and TETtargeting miR-767 induced by DNA hypomethylation in tumors. Epigenetics 9 : 1163-1171.

13. Zhou W, Fong MY, Min Y, Somlo G, Liu L, et al. (2014) Cancer-secreted miR105 destroys vascular endothelial barriers to promote metastasis. Cancer Cell 25: $501-515$ 